“Transfer” XV: 1-2 (2020), pp. 295-311. ISSN: 1886-554

\title{
LA DEDICACIÓN INFATIGABLE DE ANNA VERTUA GENTILE COMO EDUCADORA Y ESCRITORA
}

\author{
Salvatore Bartolotta (ORCID: 0000-0002-2386-5408) - \\ María Gracia Moreno Celeghin (ORCID: 0000-0001-6086-0983) \\ Universidad Nacional de Educación a Distancia (UNED)
}

\begin{abstract}
Anna Vertua nació en $1845^{1}$ en Dongo, en la provincia de Como, una localidad a la que su padre, Rocco Vertua, se había trasladado por motivos de trabajo y en la que conoció a Ester Polti, con quien contrajo matrimonio en 1844. Allí transcurrió su infancia y adolescencia, hasta que, con 17 años, tras el fallecimiento de su padre, inició a ejercer como maestra en Vicenza en el Istituto delle Dame inglesi, donde enseñó lengua y literatura. Fue precisamente en el ámbito de la pedagogía en el que se encuadran sus primeras obras, como Letture educative per fanciulle (1868), firmándolas como Annetta Vertua; de hecho, toda su producción literaria, que fue vastísima (más de 150 obras) está impulsada por una motivación educadora, con el fin de llevar sus escritos a las jóvenes italianas que nacieron al mismo tiempo que Italia como nación. ${ }^{2}$
\end{abstract}

Es preciso señalar que, a finales del siglo XIX, en una Europa que se tambaleaba por las revoluciones liberales que agitaron sus cimientos pluriseculares, de las nuevas corrientes procedentes de los idearios progresistas del positivismo surgió la necesitad natural de resquebrajar los profundos fundamentos tradicionales dictados por la jerarquización masculina en todos los sectores públicos y privados de la sociedad. Muchas fueron las voces, non solo femeninas, que denunciaron el papel secundario que ejercía la mujer en la sociedad. Una de las más relevantes, fue la del filósofo y economista inglés John Stuart Mill (1806-1873), quien de-

\footnotetext{
${ }^{1}$ Aunque la mayoría de los estudiosos datan la fecha de su nacimiento en 1850 , Angelo Cerizza, en su artículo Anna Vertua Gentile (2013), la sitúa en 1845.

${ }^{2}$ Es pertinente recordar que el Reino di Italia fue constituido el 17 de marzo de 1861 tras el período conocido como Risorgimento iniciado a principios del siglo XIX y constituido por una serie de acciones políticas y bélicas que condujeron a la unificación italiana.
} 
“Transfer” XV: 1-2 (2020), pp. 295-311. ISSN: 1886-554

nunció la injusta distribución de los roles masculinos y femeninos que relegaban a la mujer al ámbito doméstico (como madres, esposas, hijas o hermanas).

En los años en los que vivió Annetta, las profesiones a las que se les permitía el acceso a la mujer eran aquellas en las que podía poner en práctica las "naturales" capacidades que ejercía en el hogar cuando educaba con paciencia y sensibilidad a sus hijos, se ocupaba con cariño de su marido y los cuidaba con dedicación cuando estaban enfermos; en efecto, los campos de la educación y de la medicina fueron los primeros que abrieron sus puertas a la mujer como figura profesional. (Bartolotta \& Moreno 2019: 21)

La situación de la mujer en Italia no variaba mucho de la de sus congéneres europeas, con la diferencia de que este país sufriría, en el segundo cuarto del siglo XIX, una auténtica revolución, no solo política e histórica, sino administrativa y social. La construcción de una nación exigía afrontar una nueva distribución a nivel administrativo, educativo, político y local, y las profundas reformas que hubo que abordar reflejan fielmente el pensamiento atribuido al estadista Massimo D'Azeglio "Fatta l'Italia, bisogna fare gli italiani".

Había que formar a los italianos... y a las italianas; ${ }^{3}$ y uno de los medios más efectivos para formar a los nuevos ciudadanos era y es, sin duda, la labor educativa llevada a cabo en las escuelas. Sin embargo, las primeras leyes ${ }^{4}$ que se aprobaron para reformar el sistema educativo de la nueva nación adolecieron de un claro corte patriarcal que perpetuaba la estructura tradicional y el reparto de roles masculinos y femeninos. Fueron los maestros y las maestras de aquellos primeros años de la Italia unificada quienes, mal pagados y con escasa formación, se convirtieron en los

\footnotetext{
${ }^{3}$ En 1861 el $84 \%$ de las mujeres eran analfabetas, frente al $71 \%$ de los hombres (Cagnolati 2014:19).

${ }^{4}$ En los años anteriores a la unificación, se multiplicaron los debates sobre la educación, no solo de las mujeres, sino también de las clases populares, quienes se veían privadas de una formación básica. La Ley Casati, aprobada en el Piamonte de los Saboya mediante Real Decreto el 13 de noviembre de 1859 y aplicada en el Reino de Italia, palió en parte con esa situación.
} 
“Transfer” XV: 1-2 (2020), pp. 295-311. ISSN: 1886-554

transmisores del espíritu nacional que se requería en los futuros adultos. Ellos y ellas, los maestros y las maestras, debían ser modelo de esos valores que la clase dirigente pretendía difundir. ${ }^{5}$

En este contexto Anna Vertua dio sus primeros pasos como maestra y escritora. Tuvo la fortuna de crecer en una familia de clase media, y en contacto con el ambiente de renovación que se vivía; algunos de sus miembros manifestaron vivos sentimientos de fervor patriótico, como el abuelo Antonio, un jacobino masón, y su tía Julieta, la primera que agitó la bandera tricolor en su localidad natal, Codogno, de donde procedía su familia. El marido de Anna, Iginio Gentile, profesor de profesor de Historia Antigua en la Universidad de Pavía y escritor de destacadas obras de ensayo, era un patriota convencido y fue quien animó a Annetta a dedicarse a la literatura y la introdujo en el ambiente intelectual y editorial de Pavía y de Milán, ciudades en las que se estableció el matrimonio y en las que se vivieron con especial intensidad los numerosos episodios patrióticos de aquellos años, así como los movimientos en favor de los derechos de las mujeres en materia política y educativa.

Respecto al feminismo, Anna Vertua mantuvo siempre una posición moderada, sin llegar a extremismos ni radicalismos, como manifiesta en todas sus obras, en las que las protagonistas femeninas desarrollan sus potenciales cualidades dentro del canon tradicional (Bartolotta \& Moreno 2019).

Sin embargo, no fue ajena a la oleada de reivindicaciones que permeaban la sociedad italiana respecto a, sobre todo, el derecho a la educación de las mujeres. De hecho, participó en Milán en

\footnotetext{
${ }^{5}$ En especial, a las maestras se les exigía que perpetuaran el orden moral y los valores tradicionales de la sociedad: “All'indomani dell'Unità, quando la nascita della scuola elementare pubblica e l'istituzione dell'obbligo scolastico resero necessaria e urgente la formazione di una nuova classe magistrale, il suo incremento numerico e culturale, la «donna» apparve subito al ceto dirigente di allora e agli intellettuali attenti alle questioni scolastiche, il soggetto naturalmente destinatario di una missione rivolta all'alfabetizzazione dei ceti popolari, in una prospettiva di salvaguardia dell'ordine morale e delle gerarchie sociali. La vocazione magistrale venne descritta con grande enfasi e le fu attribuita la stessa sacralità del ruolo materno". (Covato 2012: 167)
} 
“Transfer” XV: 1-2 (2020), pp. 295-311. ISSN: 1886-554

el Congreso sobre los derechos de la mujer celebrado en $1907 .{ }^{6} \mathrm{La}$ educación femenina moderna fue un camino arduo y lento y se alcanzaron objetivos a la par que avanzaban los movimientos liberales y democráticos fomentados por las voces de hombres y mujeres que dedicaron sus esfuerzos en lograr una educación paritaria. ${ }^{7}$ Y Anna Vertua fue una gran defensora de la instrucción femenina, con el fin de que la mujer fuera capaz de explotar sus dotes y conocimientos para el servicio de la sociedad así como en el hogar, como esposa y madre. Implicada en el fervor patriótico que se respiraba en la Italia recién creada, nuestra autora pensaba que a través de la educación los ciudadanos se convertirían en verdaderos italianos.

Ella amaba enseñar y siempre se consideró una educadora que tuvo la oportunidad de transmitir a través de sus obras, tanto sus conocimientos pedagógicos (como los relatos para niños y niñas, lecturas para manuales educativos, obras de teatro infantiles, cuentos de marionetas), ${ }^{8}$ como sus ideas sobre el mundo femenino, en especial sobre cómo las jóvenes podían desarrollar su potencial dentro del hogar y fuera de él. Ya en los títulos de muchas de sus obras, en especial de los manuales de comportamiento se aprecia la intención educativa de Vertua con el fin de que las jóvenes lectoras aprendieran cómo comportarse en casa y

\footnotetext{
${ }^{6}$ En el Congreso, uno de los primeros que se celebraron en Italia, se debatió también sobre otros temas, como el del voto femenino. Participaron destacadas representantes de la sociedad italiana como Maria Montessori, Teresa Labriola, Anna Maria Mozzoni o Emilia Mariani (Filippini 2006).

${ }^{7}$ Estamos hablando de figuras como Anna Maria Mozzoni, Gualberta Alaide Beccari, Gugliemina Ronconi, y escritoras como Sofia Bisi Albini, Ada Negri o Flavia Steno, entre muchas otras.

${ }^{8}$ La mayor parte de ellos los escribió antes de fin de siglo y destacamos: Letizia e Sandro: racconto per fanciulli (1875); Quadretti di storia naturale per il mio fanciullo (1883); Oh bei! oh bei!: balocchi e fiabe per bambini (1885); Ricreazioni e feste: commediole, monologhi dialoghi d'occasione per istituti e scuole maschili e femminili; So leggere: libro per l'infanzia; Mi diverto a leggere: libro per l'infanzia; Comincio a leggere: libro per l'infanzia (1895); Cassetta misteriosa: scene familiari per fanciulle; Cuor forte e gentile: racconto per giovanette; Il pesce cane: scene famigliari per fanciulle; Le feste della fanciullezza: racconti (1898); In vacanza e vari altri raccontini (1899); Amico sincero ed altri raccontini (1900); Un' allegra nidiata: libro di lettura per la quarta classe elementare femminile (1902).
} 
“Transfer” XV: 1-2 (2020), pp. 295-311. ISSN: 1886-554

en sociedad. Este género literario abarca breves ensayos en la misma línea que los de galateo que estaban dirigidos a un público joven, en especial femenino, con el fin de instruirlo con las buenas costumbres preestablecidas. De hecho, buena parte de su obra pertenece a este género literario tan de moda en aquella época y la mayoría de las escritoras de finales del siglo XIX cuentan con uno en su haber. Entre todos los que escribió, ${ }^{9}$ destaca Come devo comportarmi?: libro per tutti, publicado en 1897 por la editorial Hoepli, un manual que obtuvo un gran éxito de público, con once ediciones y varias reediciones y del cual existe una versión adaptada y traducida en español por Pedro J. Llort, destinada al público hispanoamericano. ${ }^{10}$ En él, aunque el título incluya un hipotético "todos", Vertua se dirige a las jóvenes y les da consejos sobre cuáles son las normas que deben seguir para comportarse en sociedad, siguiendo la senda de la virtud, de la discreción y la laboriosidad. Sin embargo, manifiesta en esta obra, así como en muchas otras, su posición aperturista hacia la emancipación de la mujer que no tiene que por qué estar permanentemente acompañada y protegida. Véase en estas líneas cómo, pese a las reticencias de muchos, la autora rompe una lanza en favor de un retazo de la ansiada libertad que abriera las puertas de la jaula de cristal en la que vivían las mujeres prisioneras de sus padres, hermanos o maridos, y afirma que no es tan indecoroso o peligros dejar salir a las jóve-

\footnotetext{
${ }^{9}$ Entre la producción de Anna Vertua encontramos, entre otros, estos manuales de comportamiento son: L'arte di farsi amare dal marito: consigli alle giovani spose (1889); Per la mamma educatrice (1894); Per esser garbate: piccolo galateo delle fanciulle (1899); Voce dell'esperienza: libro per le signorine (1899); Voce materna: consigli ed esempi alle madri e alle giovanette (1903); La potenza della bontà: libro per le signorine (1905); A te, sposa (1919).

${ }^{10}$. Esta es parte de la reseña publicada por semanario "La Unión", en su número del 27 de febrero de 1930: "Las personas que tengan interés en destacar por su educación y simpatía personal; que quieran aprender y practicar el trato social y familiar correcto; que aspiren a triunfar y a alcanzar la felicidad para su hogar, sean del sexo que sean, encontrarán en ¿Cómo debo comportarme? el guía seguro y eficaz que de una manera agradable y amena les conducirá a la meta de sus propósitos. ¿Cómo debo comportarme? es un libro moral que puede y debe ponerse en todas las manos. Sacarán de su lectura enseñanzas provechosas tanto los padres como los hijos, recomendándose especialmente a los jóvenes y doncellas en vísperas de contraer matrimonio" (La Unión, 27 de febrero de 1930: 3)
} 
“Transfer” XV: 1-2 (2020), pp. 295-311. ISSN: 1886-554

nes $^{11}$ "cuando se trate de algún recado o de visitas entre amigas íntimas o familiares" (T. de los A.):

...ora in alcune famiglie è entrata l'idea di lasciar uscire sole le signorine quando si tratti di qualche commissione o di visite fra amiche intime o parenti stretti, non è poi quella cosa indecorosa e pericolosa che si credeva. (Vertua Gentile 1897a: 151)

Aunque no se trata de un manual en el sentido estricto de la palabra, Romanzo d'una signorina per bene, publicado en el mismo año, nos presenta las cualidades de la protagonista Lucia (virtuosa, culta, discreta, obediente hacia su progenitor, al que adora tras quedarse huérfana) y relata las vicisitudes de la joven que debe enfrentarse a la ruina del patrimonio familiar y que, con gran entereza, saca fuerzas de la debilidad y supera la ahogada situación en la que se encuentra hasta encontrar el amor, justo premio a su virtud. En el siguiente fragmento, Vertua, en boca de la protagonista, alaba la indiferencia de las jóvenes a las que no les afectan las lisonjas ni las palabras vanas ni las atenciones de quienes ven solo en ellas unas "muñecas" sin considerar su ingenio y sus sentímientos. Este fragmento es uno de los tantos ejemplos en los que la educadora/escritora anima a las jóvenes a huir de esos clichés, a potenciar sus capacidades intelectuales y a cultivar sus sentímientos... Nos encontramos a finales del siglo XIX; sin embargo, se trata de enseñanzas plenamente válidas hoy en día:

Oh! gran sfortuna, mia cara, avere l'anima fatta in maniera, che i complimenti vi scivolano sopra senza lasciarvi traccia, anzi recandovi una spiacevole sensazione di freddo! ... Gran sfortuna non credere alle paroline melate, alle occhiate, come si dice, assassine; e vedere, là, nettamente, come bersaglio a vivaci colori, la mira cui tendono i molti vagheggini, complimentosi fino a l'insolenza, cor-

\footnotetext{
${ }^{11}$ Vertua recoge los avances que, poco a poco, se lograban en materia de libertad de movimientos por parte de las mujeres en la Italia de finales de siglo. Y, de acuerdo con Verdirame, las autoras de obras catalogadas como manuales de comportamiento, compartían los anhelos de libertad que todas sentían, eso sí, advirtiendo de los "peligros" que estaban al acecho en el mundo fuera de las paredes domésticas (Verdirame 2009: 100).
} 
“Transfer” XV: 1-2 (2020), pp. 295-311. ISSN: 1886-554

teggiatori fino a l'offesa, che hanno l'aria di averti in conto di bambola senza intelligenza né sentimento, e così sciocca da essere lusingata dalle loro litanie di menzogne, colpita e tocca da svenevolezze ridicole e oltraggianti! (Vertua Gentile 1897b: 50-51) ${ }^{12}$

A quince años de distancia, Vertua relata la fortaleza de otra joven, Carla, que emerge como paladina de la honestidad y del amor abnegado, protagonista femenina de la novela Carlo e Carla: romanzo (1913), quien sostiene a su amado en los momentos de incertidumbre sin caer en el desánimo. En el fragmento escogido, Vertua pone en boca de uno de los compañeros de Carlo la admiración que suscita entre todos, la virtud, la integridad, la cordialidad discreta y elegante de su joven amiga. Vertua muestra en estas páginas a sus jóvenes lectoras cuáles son las cualidades que debe admirar un hombre en una mujer, más allá de su belleza: ${ }^{13}$

- [...]. Fin da ragazzina, che portava le sottane corte e la treccia spiovente, era indulgente, gentile, senza affettazione, compiacentissima. Mai che nessuno le abbia usato uno sgarbo o le abbia mancato di rispetto! ... Come adesso, tale e quale, che tutti le vogliono bene e la rispettano come una sorella. Ha sempre avuto una maniera tutta sua da impedire che in sua presenza si dicessero parole troppo vibrate o si usassero modi non perfettamente corretti. La sua presenza nella scuola ci ha fatto un gran bene a noi ragazzacci; e questo senza che noi ce ne accorgessimo e che lei capisse. Era il nostro freno e il nostro modello. (Vertua Gentile 1922: $11)^{14}$

\footnotetext{
12 “¡Oh! ¡Qué mala suerte, querida, tener el alma hecha de manera que los cumplidos os resbalan sin dejar huella, es más, dándoos una desagradable sensación de frío! jQué mala suerte no creer en las palabras melosas, en las ojeadas, cómo se dice, asesinas; y ver, allí, con nitidez, como diana de vivos colores, el objetivo al que apuntan los muchos admiradores, obsequiosos hasta la insolencia, pretendientes hasta la ofensa, que parece que te consideran como a una muñeca sin inteligencia ni sentimientos, y tan tonta que te sientes halagada por sus letanía de falsedades, tocada y golpeada por cursilerías ridículas y ultrajantes!” (T. de los A.). ${ }^{13}$ La edición consultada es la de 1922.

14 “- [...]. Desde que era una chiquilla, cuando llevaba la falda corta y la trenza sin recoger, era comprensiva amable, nada artificial, siempre dispuesta. iJamás nadie la despreció o le faltó el respeto! ... Tal y como ahora, que todos la quieren y la respetan como una hermana. Siempre ha tenido unas maneras tales que hacían
} 
“Transfer” XV: 1-2 (2020), pp. 295-311. ISSN: 1886-554

Fueron muchas las vicisitudes que marcaron la existencia de esta trabajadora infatigable, y autora de más de ciento cincuenta títulos, en especial las dos muertes que marcaron su vida, la de su marido en 1893, pero sobre todo la de su hijo Marco Tullio en 1912. Escribió incansablemente, no solo como autora de relatos, novelas y manuales, sino también como colaboradora en publicaciones periódicas y fundadora de revistas, como hicieron muchas de sus coetáneas. Fue una autora muy conocida en su época y sus obras gozaron de gran difusión, aunque no lograría alcance internacional a través de traducciones, a excepción del ya mencionado Come devo comportarmi?

Destaca su colaboración en Italia Giovane, de 1891 a 1896, o en Cordelia ${ }^{15}$ y llegó a dirigir en 1905 Fanciullezza italiana. ${ }^{16}$

Su colaboración en Il secolo XIX nella vita e nella cultura dei popoli $^{17}$, con el ensayo Vita Intima ${ }^{18}$ fue especialmente interesante porque, sin excederse de la posición moderada con respecto a las reivindicaciones femeninas, Vertua se manifiesta abiertamente feminista al defender la liberación de la mujer a nivel no solo personal y familiar, sino social, económico y jurídico. La publicación, de carácter divulgativo, tenía como objetivo ser una guía educativa destinada a los jóvenes miembros de la familia. La publicación es uno de los innumerables ejemplos de ese filón que tanto éxito

que, en su presencia, no se dijeran palabras fuera de tono o se usaran malos modos. Su presencia en el colegio nos ha hecho mucho bien a nosotros, a los chicos; y sin que nos diéramos cuenta ni que ella lo hiciera aposta. Era nuestro freno y nuestro modelo". (T. de los A.).

${ }^{15}$ Cordelia. Foglio settimanale per le giovinette italiane, fue fundado por Angelo De Gubernatis en 1881 fue la primera de las publicaciones dirigidas a las jovencitas publicada en Italia.

${ }^{16}$ La publicación (1904-1912) estaba dirigida a los más pequeños y contenía ilustraciones con el fin de proporcionarles enseñanzas de manera lúdica. En ella colaboró la escritora y también maestra Sofia Bisi Albini (1856-1919).

${ }^{17}$ No es posible determinar la fecha exacta del volumen de la publicación; seguramente después de 1902.

${ }^{18}$ En el mismo número, además de Anna Vertua con su sección sobre la religión y la familia, colaboraron Mara Antelling, Silvio Zambaldi y Scipio Sighele, que ofrecían consejos y sugerencias, sobre la moda y el deporte y la vida social. 
“Transfer” XV: 1-2 (2020), pp. 295-311. ISSN: 1886-554

tuvo entre la media y alta sociedad, cuyos editores, directores y colaboradores, se propusieron difundir normas de comportamiento, novedades en moda y peinados, y también breves recensiones de obras de poesía, teatro y narrativa de autores contemporáneos (Cagnolati 2014).

El ensayo elaborado por Anna Vertua, Vita intima (pp. 3-80) está dividido en siete capítulos, agrupados según las líneas temáticas a desarrollar: la mujer y el hombre, los distintos roles en la familia (Cap. I); los cuidados del cuerpo y de la mente (Cap. II); las clases pobres y los cambios en la sociedad (Cap. III); la filantropía y la beneficencia (Cap. IV); la educación y la escuela (Cap. V); religión y religiones (Cap. VI); la mujer trabajadora y el matrimonio (Cap. VII). La lectura de algunos de sus párrafos permite tener una visión de primera mano sobre cómo estaba estructurada la sociedad de la Italia de principios de siglo y por cuáles normas estaba regida; al mismo tiempo, Anna Vertua, al manifestar su posición sobre ámbitos tan intocables como la religión, la familia o los roles del hombre y de la mujer, abre fisuras por las que dejar pasar las nuevas ideas que se respiraban en Europa y en E.E.U.U., y difundirlas entre sus jóvenes lectores y lectoras.

En primer lugar, la sociedad moderna no necesita mujeres pasivas, sometidas al hombre patriarca, sino compañeras que, conservando su dulzura y sensibilidad, sean inteligentes y cultas para construir un futuro juntos:

Questo ora l'uomo sente e capisce e vuole nella donna non la schiava, no l'idolo, non la serva ma la compagna. La compagna capace di comprenderlo, di esercitare una benefica influenza su la sua intelligenza e la sua energia: capace di sostenerlo del suo coraggio, fatto di amore e di annegazione, nei momenti di sconforto; capace di ricevere nel suo cuore, sempre aperto, ogni intimo sfogo, e di confortare il dolore del dubbio, l'angoscia del malcontento, lo strazio della delusione... L'uomo di adesso sa valutare i pregi della donna e trova in essa valido aiuto, consigli, incoraggiamenti, riposo. (Vertua Gentile 1902: 4-5) ${ }^{19}$

\footnotetext{
19 "Esto el hombre ahora lo siente y lo entiende, y quiere una mujer que no sea esclava, que no sea un ídolo, que no sea una sierva, sino una compañera. Una
} 
“Transfer” XV: 1-2 (2020), pp. 295-311. ISSN: 1886-554

Vertua, a lo largo de estas páginas, sostiene (a menudo apasionadamente) que la principal causa del nuevo empuje de la mujer en la familia y en la sociedad, llega a través de la educación:

Una di queste cause, molti la riconoscono nella grande riforma educatrice della donna; riforma che ha per fondamento e per conseguenza la giustizia e soprattutto la stima dell'uomo per la donna stimata nel suo giusto valore, libera da l'oppressione che le imprigionava le facoltà giacenti inerti, indistinte, abbuiate, sente in tutta la sua forza la propria individualità, e l'anima sua, fatta di sentimento, al sentimento si abbandona. (Vertua Gentile 1902: 11-12) ${ }^{20}$

Solo si la mujer accede a la educación, podrá formar y completar su personalidad con el fin de ascender en su propio status como esposa y como madre en el ámbito familiar "ove la tenerezza avvicina e la mutua simpatia intellettuale interessa ed avvince; la famiglia retta e guidata dalla madre che può e vuole essere la prima educatrice dei figli suoi" (Vertua Gentile 1902: 12). ${ }^{21}$ Sin embargo, buena parte de sus convicciones sobre la educación femenina, tienen como fin último la emancipación de la mujer a través de un trabajo remunerado que, o bien, les permita ser completamente independientes, o que contribuya a mantener a la familia. A pesar

compañera capaz de comprenderlo, de ejercer una benéfica influencia sobre su inteligencia y su energía: capaza de sostenerlo gracias a su coraje, hecho de amor y de abnegación, en los momentos de desánimo; capaz de recibir en su corazón, siempre abierto, cualquier íntimo desahogo, y de consolar el dolor causado por la duda, la angustia de la tristeza, el desgarro de la desilusión... El hombre actual sabe valorar las virtudes de la mujer, y encuentra en ella una ayuda válida, consejos, aliento, reposo" (T. de los A.).

${ }_{20}$ "Una de estas causas, muchos la reconocen en la gran reforma educadora de la mujer; reforma que tiene por fundamento y consecuencia la justicia y, sobre todo, la estima del hombre hacia la mujer apreciada en su justo valor, libre de la opresión que le apresaba las facultades que yacían inertes, confundidas, oscurecidas, que siente su propia individualidad con toda su fuerza y, su alma, hecha de sentimiento, al sentimiento se abandona" (T. de los A.).

${ }_{21}$ "donde la ternura acerca, y la mutua simpatía intelectual interesa y atrae; la familia dirigida y guiada por la madre que puede y quiere ser la primera educadora de sus hijos" (T. de los A.). 
“Transfer” XV: 1-2 (2020), pp. 295-311. ISSN: 1886-554

de que Vertua defiende a capa y espada el trabajo femenino, nos sorprende al destacar los inconvenientes que acarrea y nos recuerda que no es tanta la distancia que nos separa de aquellas madres trabajadoras de finales del siglo XIX. ${ }^{22}$ En cualquier caso, los avances logrados no deben borrarse solo por las consecuencias logísticas que comporta el hecho de que la mujer trabaje fuera del hogar, a lo que Vertua se echa las manos a la cabeza:

Per molte donne è necessario lavorare per aiutare il marito nel sostentamento della famiglia. Per moltissime è indispensabile il lavoro, che le rende indipendenti, che le toglie a le mortificazioni di essere d'aggravio a la famiglia.

Sicuro, la donna dovrebbe essere l'angelo della casa e non occuparsi che di essa e dei figliuoli. Il bellissimo libro di Jules Simon su l'operaia, mira appunto a questa conclusione: l'uomo solo dovrebbe uscire dalla casa per guadagnare il pane della famiglia; ma è questa una idealità che per ora è ben lontana dall'essere realizzata. E forse chi sa?... il sogno che a taluni può apparire bello, avrebbe per fine di ripiombare la donna nell'antico stato dal quale si è elevata con tanto stento e in sì lungo seguito di anni! (Vertua Gentile 1902: 74$)^{23}$

\footnotetext{
${ }^{22}$ Hablando de la madre trabajadora, enumera las tareas que la esperan al acabar la jornada laboral: recoger a los pequeños, ocuparse de la casa, preparar la cena: "Ed è quella, ordinariamente la sola ora in cui i membri della famiglia si trovano; ora in cui la stanchezza, che non può avere un pronto conforto di riposo, spesso si sfoga in mali umori, in rimbrotti, in lagnanze e peggio" (Vertua Gentile 1902: 73). < "Y esa es, por lo general, la única hora en la que los miembros de la familia se encuentran: la hora en la que el cansancio, que no puede tener un inmediato alivio en el descanso, a menudo se desahoga en males humores, reproches, laméntelas y peor" (T. de los A.).

${ }_{23}$ "Para muchas mujeres es necesario trabajar para ayudar a sus maridos a mantener a la familia. Para muchísimas es indispensable el trabajo que las hace independientes, que las despoja de la mortificación de ser un peso para la familia.

Seguro, la mujer debería ser el ángel de la casa y no ocuparse más que de ella y de los hijos. El precioso libro de Jules Simon sobre la obrera apunta, precisamente, a esta conclusión: solo el hombre debería salir de casa para ganar el pan de la familia; pero este es un ideal que, de momento, está muy lejos de cumplirse. Y, quizás ¿quién sabe? El sueño que a algunos puede parecerle bello, tendría como fin volver a hacer caer a la mujer en el antiguo estado del que se ha elevado con tanto esfuerzo ¡y a lo largo de tantos años!” (T. de los A.).
} 
“Transfer” XV: 1-2 (2020), pp. 295-311. ISSN: 1886-554

En lo relativo a la educación, Vertua muestra una visión moderna y rabiosamente actual, reflejada en su convicción de que la escuela debe ser el lugar en el que los niños y las niñas aprendan no solo conocimientos en historia, aritmética, geografía o gramática, sino también una educación moral y social cuya enseñanza estaba antiguamente en manos de las autoridades religiosas:

Si potrebbe introdurre nell'insegnamento un'unità di spirito, uno scopo, dandogli un'orientazione sociale e morale, facendo convergere tutto a la formazione dell'uomo e del cittadino. (Vertua Gentile 1902: 56$)^{24}$

Como si de una misión se tratara, nos encontramos con la necesidad de formar a los futuros ciudadanos y ciudadanas de la nueva nación.

De la lectura de las páginas de este pequeño manual se desprende un retrato fiel de la sociedad italiana, de los problemas reales que tenían que afrontar y superar las familias, en particular las mujeres esposas, madres y trabajadoras. Problemas que no distan mucho de los actuales. Anna Vertua, ella misma, esposa, madre y trabajadora infatigable, educadora por formación y vocación, nos recuerda que garantizar el acceso a la educación es imprescindible para que las mujeres logren liberarse de los antiguos vínculos que las tiene recluidas y que les impiden caminar por sí mismas, adoptar sus propios criterios, desarrollar su inteligencia, su gran capacidad de trabajo y sus cualidades. Gracias a ella, y a otras autoras de su tiempo, las mujeres italianas de finales del siglo XIX y principios del XX tuvieron una guía que les indicaba el camino que debían recorrer. Un camino que llega hasta nuestros días.

\footnotetext{
24 "Se podría introducir en la enseñanza una unidad de espíritu, una finalidad, dándole una orientación social y moral, haciendo que todo convergiera en la formación del hombre y del ciudadano" (T. de los A.).
} 
“Transfer” XV: 1-2 (2020), pp. 295-311. ISSN: 1886-554

\section{Referencias bibliográficas}

ANDRIANOPOLI CARDULLO, Mariacarla. (1977). Letture e modelli comportamentali: motivi moralistici ed esemplaristici nei libri italiani di lettura per l'infanzia, la fanciullezza e le famiglie (1830-1914). Génova: Tilgher.

BARTOLOTTA, Salvatore \& MORENO CELEGHIN, Mª Gracia. (2019). "Los géneros de Annetta Vertua". En: HERNÁNDEZ ÁLVAREZ, M ${ }^{a}$ Vicenta. (Coord.), Escritoras de la Modernidad (1880-1920). La transformación del canon. Granada: Editorial Comares, pp. 17-24.

BERNARDINI NAPOLETANO, Francesca. (1998). "Scritture femminili per l'infanzia tra Ottocento e Novecento". En: AA.VV. Inchiostri per l'infanzia: letteratura ed editoria in Italia dal 1880 al 1965. Roma: De Luca, pp. 13-19.

BLEZZA, Silvia. (2004). Libri, bambini, ragazzi: incontri tra educazione e letteratura. Milán: Vita e Pensiero.

BOERO, Pino \& DE LUCA, Carmine. (1995). La letteratura per l'infanzia. Roma: Laterza.

BUCK, Clare. (1992). Bloomsbury Guide to Women's Literature. Londres: Bloomsbury Publishing.

CAGNOLATI, Antonella. (2014). "Donne, maestre, giornaliste: la stampa pedagogica all'indomani dell'Unità d'Italia (18611865)." Educació i Història: revista d'història de l'educació, 2014:. 17-27.

CERIZZA, Angelo. (2013). "Anna Vertua Gentile, Scrittrice". En: CAVALLERA, Hervé A. \& SCANCARELLO, Walter. (ed.). Scrittrici Italiane dell'Otto e Novecento, le interviste impossibili. Siena: Bibliografia e informazione, pp. 79-89.

COPPOLA, Goffredo; CALOGERO, Guido \& CAPODAGLIO, Giulio (1934). "Stuart Mill”. Enciclopedia Italiana. Recuperado de: $<<$ http://www.treccani.it/enciclopedia/john-stuartmill_\%28Enciclopedia-Italiana\%29/>> (Fecha última consulta 30-08-2019).

COVATO, Carmela. (2012). "Maestre d'Italia. Uno sguardo sull'età liberale." Storia delle donne, 8(1): 165-184. Recuperado de: 
“Transfer” XV: 1-2 (2020), pp. 295-311. ISSN: 1886-554

$<<$ http://www.storiadelledonne.it/wpcontent/uploads/2009/03/covato2012.pdf $>>$ (Fecha última consulta 30-08-2019).

CUTRONA, Federica. (2002). "Il palcoscenico dei buoni sentimenti: Anna Vertua Gentile scrittrice di teatro per l'infanzia." En: BOERO, Pino. (ed.). Storie di donne: Contessa Lara, Anna Vertua Gentile, Ida Baccini, Jolanda: scrittura per l'infanzia e letteratura popolare fra Otto e Novecento. Génova: Brigati, pp. 35-44.

D'AGOSTINO, Domenico. (2010). "Escritoras italianas para la infancia”. En: GONZÁLEZ DE SANDE, Estela. (ed.). Rebeldes literarias. Sevilla: ArCiBel Editores, pp. 267-280.

DUGGAN, Christopher. (1994). A Concise History of Italy. Cambridge-Nueva York: Cambridge University Press.

FILIPPINI, Nadia Maria. (ed.). (2006). Donne sulla scena pubblica: società e politica in Veneto tra Sette e Ottocento (vol. 349). Milán: Franco Angeli.

FRANCHINI, Silvia \& SOLDANI, Simonetta. (ed.) (2004). Donne e giornalismo: percorsi e presenze di una storia di genere. Milano: Franco Angeli.

GONZÁLEZ DE SANDE, Estela. (2010). Rebeldes literarias. Sevilla: ArCiBel Editores.

JONES, Verina R. \& LEPSCHY, Anna Laura. (2000). With a Pen in Her Hand: Women and Writing in Italy in the Nineteenth Century and Beyond. Exeter: Society for Italian Studies. La Unión, $\mathrm{n}^{\mathbf{0}} 1226$ (27 de febrero de 1930), Jaca.

RE, Lucia. (2001). "Passion and Sexual Difference: The Risorgimento and the Gendering of Writing in Nineteenth-Century Italian Culture". En: RUSSELL ASCOLI, Albert \& VON HENNEBERG, Krystyna. (ed.). Making and Remaking Italy: The Culti-vation of National Identity around the Risorgimento. Nueva York: Berg, pp. 155-200.

SPINAZZOLA, Vittorio. (1997). Pinocchio \& C.: La grande narrativa italiana per ragazzi. Milán: Il saggiatore.

TURNATURI, Gabriella. (2011). Signore e signori d'Italia: una storia delle buone maniere. Milán: Feltrinelli Editore. 
“Transfer” XV: 1-2 (2020), pp. 295-311. ISSN: 1886-554

VERDIRAME, Rita. (2009). Narratrici e lettrici (1850-1950): le letture della nonna dalla Contessa Lara a Luciana Peverelli: con testi rari e documenti inediti. Padova: libreriauniversitaria.it Editore.

VERTUA GENTILE, Anna. (1897a). Come devo comportarmi?: libro per tutti. Milano: U. Hoepli (tr.es. ¿Cómo debo comportarme?: Libro Para Todos de la duodécima edición italiana, modernizada y puesta al día por Lidia Morelli; traducción y adaptación de Pedro J. Llort).

---. (1897b). Romanzo di una signorina per bene. Milano: Paolo Carrara Editore. Recuperado de:

$<<$ https://www.gutenberg.org/files/22507/22507-pageimages $/>>$ (Fecha última consulta 30-08-2019).

---. (1902). "Vita intima". En: Il secolo XIX nella vita e nella cultura dei popoli, vol. XVII, Milán: Vallardi. Recuperado de: <<https://archive.org/details/digitami_TO00853301>> (Fecha última consulta 30-08-2019).

---. (1922). Carlo e Carla. Milano: Barion. Ed. Recuperado de: <<https://www.liberliber.it/mediateca/libri/v/vertua/carlo e_carla/pdf/carlo__p.pdf $>>$ (Fecha última consulta 30-082019).

WOOD, Sharon. (1995). "Unification: Making and Unmaking the Nation." Italian Women's Writing 1860-1994. Nueva Jersey: Athlone, pp. 3-25.

Fecha de recepción: 2.09.2019 Fecha de aceptación: 01.10.2019 
“Transfer” XV: 1-2 (2020), pp. 295-311. ISSN: 1886-554

\begin{abstract}
Resumen:
Anna Vertua Gentile fue maestra y escritora en una época fundamental en la historia de Italia, puesto que vivió a caballo de los siglos XIX y XX siendo testigo directo de los cambios políticos, sociales y culturales en los que se vio sumergido el país antes y después de su unificación como Reino. Fue una de las autoras más productivas e infatigables del momento y gozó de un gran éxito, cultivando diferentes géneros literarios: relatos y cuentos para niños y niñas, obras de teatro infantiles, además de los llamados "manuales de comportamiento", dirigidos en especial al público femenino con un fin claramente pedagógico y educativo. Sin embargo, como tantas otras mujeres escritoras, fue olvidada y su obra apenas fue traducida. Con este artículo pretendemos rescatar su figura con el fin de que sea justamente reconocida.
\end{abstract}

Palabras clave: Vertua Gentile, Manuales de comportamiento, Educación, Traducción.

\title{
ANNA VERTUA GENTILE'S INDEFATIGABLE DEDICATION AS EDUCATOR AND WOMEN WRITER
}

\begin{abstract}
:
Anna Vertua Gentile was a teacher and writer at a fundamental time in the history of Italy, since she lived through the nineteenth and twentieth centuries as a direct witness of the political, social and cultural changes in which the country was submerged before and after of its unification as Kingdom. She was one of the most productive and indefatigable authors of the moment and enjoyed great success, cultivating different literary genres: stories for boys and girls, children's plays, in addition to the so-called "behavior manuals", aimed in particular at female public with a clearly pedagogical and educational purpose. However, like so many other women writers, she was forgotten and her work was hardly translated. With this article, we intend to rescue his figure in order to be justly recognized.
\end{abstract}


“Transfer” XV: 1-2 (2020), pp. 295-311. ISSN: 1886-554

Keywords: Vertua Gentile, Behavior Manuals, Education, Translation. 